\section{Peripheral Sympathetic Nerve Dysfunction in Adolescent Japanese Girls Following Immunization with the Human Papillomavirus Vaccine}

Key words: HPV vaccine, adverse event following immunization, neurological symptoms, Japan

\section{(Intern Med 54: 1953, 2015)}

(DOI: 10.2169/internalmedicine.54.4479)

To the Editor We write this letter to voice our concerns about methods used and impressions created regarding any possible relationship between the human papillomavirus (HPV) vaccine and adverse events following immunization (AEFI) in Japanese adolescent girls in the paper by Kinoshita et al. (1). We contend that the paper does not demonstrate any relationship between vaccination and adverse events, which are poorly-defined and include an eclectic range of symptoms. We feel that the paper's title and stated objective may mislead the non-expert reader to conclude that such a relationship exists. Furthermore, the assertion that vaccination is "legally required" in Japan is incorrect and may add fuel to claims by the anti-vaccine movement. To reiterate, vaccinating a child against HPV in Japan is voluntary, not mandatory.

The authors state that "a high incidence" of neurological symptoms has been found following vaccination in Japan but do not provide the evidence base or associated reference (s) to support this. They describe various syndromes and, while it may be that some cases are correctly diagnosed as Postural Orthostatic Tachycardia Syndrome (POTS) and Chronic Regional Pain Syndrome (CRPS), this does not confirm that the HPV vaccine is related to such occurrences. As described by the authors, the conditions they report peak in adolescent females and occur without HPV vaccination. To assess whether HPV vaccination is associated with disease development, one needs a robust specific case definition and demonstration of a consistent, biologically plausible temporal relationship between vaccination and the disease. In this paper we see neither. We are only told that the average time between vaccination and symptoms is 5.47 months ( \pm 5 months), thus suggesting extensive variability which argues against such a relationship.
While robust scientific methods are available for assessing vaccine-AEFI relationships (2), unfortunately they have not been used here. The authors state vaguely that they cannot conclude HPV vaccination is not associated with adverse events, but they do not acknowledge that rates may be entirely consistent with pre-vaccination occurrence. We suggest that the authors look to countries that have used existing public health infrastructure to meaningfully compare rates of neurological diseases in pre- and post-vaccination populations and have shown no association exists $(3,4)$.

Clearly, we believe and understand that the girls described in this paper are indeed suffering. However, we do not believe that this suffering has been shown to be related to HPV vaccination. We sincerely hope that the messages in this article do not prevent a highly effective (5) and safe vaccine from being provided to the young women of Japan.

\section{The authors state that they have no Conflict of Interest (COI).}

Sharon J.B. Hanley ${ }^{1}$, Kevin G.J. Pollock ${ }^{2}$ and

Kate Cuschieri ${ }^{3}$

\section{References}

1. Kinoshita T, Abe RT, Hineno A, Tsunekawa K, Nakane S, Ikeda S. Peripheral sympathetic nerve dysfunction in adolescent Japanese girls following immunization with the human papillomavirus vaccine. Intern Med 53: 2185-2200, 2014.

2. WHO, Causality Assessment of an Adverse Event Following Immunization (AEFI) [Internet]. [cited 2014 Nov. 1]. Available from: http://www.who.int/vaccine_safety/publications/gvs_aefi/en/

3. Arnheim-Dahlström L, Pasternak B, Svanström H, Sparén P, Hviid A. Autoimmune, neurological, and venous thromboembolic adverse events after immunisation of adolescent girls with quadrivalent human papillomavirus vaccine in Denmark and Sweden: cohort study. BMJ 347: f5906, 2013.

4. MHRA Public Assessment Report, Cervarix HPV vaccine: update on UK safety experience at end of 4 years use in the HPV routine immunisation programme [Internet]. [cited 2014 Nov. 1]. Available from: http://www.mhra.gov.uk/Safetyinformation/Generalsafet yinformationandadvice/Product-specificinformationandadvice/Prod uct-specificinformationandadvice-G-L/HumanpapillomavirusHPVv accine/CON023340

5. Pollock K, Kavanagh K, Potts A, et al. Reduction of low- and high-grade cervical abnormalities associated with high uptake of the HPV bivalent vaccine in Scotland. Br J Cancer 111: 18241830, 2014.

\footnotetext{
${ }^{1}$ Department of Women's Health Medicine, Hokkaido University, Japan, ${ }^{2}$ Health Protection Scotland, Scotland and ${ }^{3}$ Scottish Human Papillomavirus Reference Laboratory, Royal Infirmary of Edinburgh, Scotland

Received for publication November 10, 2014; Accepted for publication December 7, 2014

Correspondence to Dr. Sharon J.B. Hanley, sjbh1810@med.hokudai.ac.jp

(C) 2015 The Japanese Society of Internal Medicine Journal Website: http://www.naika.or.jp/imonline/index.html
} 